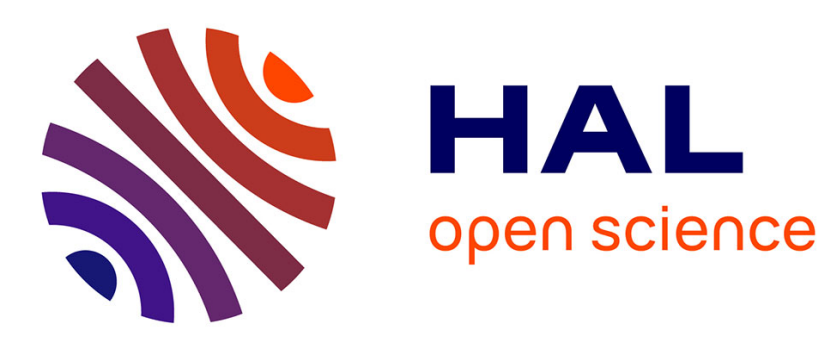

\title{
A first principle model for multiphase slugging flow in vertical risers
}

Florent Di Meglio, G.O. Kaasa, Nicolas Petit

\section{To cite this version:}

Florent Di Meglio, G.O. Kaasa, Nicolas Petit. A first principle model for multiphase slugging flow in vertical risers. 48th IEEE Conference on Decision and Control, Jan 2010, Shanghai, China. pp.8244 - 8251, 10.1109/CDC.2009.5400680 . hal-00474370

\section{HAL Id: hal-00474370 \\ https://hal-mines-paristech.archives-ouvertes.fr/hal-00474370}

Submitted on 12 May 2010

HAL is a multi-disciplinary open access archive for the deposit and dissemination of scientific research documents, whether they are published or not. The documents may come from teaching and research institutions in France or abroad, or from public or private research centers.
L'archive ouverte pluridisciplinaire HAL, est destinée au dépôt et à la diffusion de documents scientifiques de niveau recherche, publiés ou non, émanant des établissements d'enseignement et de recherche français ou étrangers, des laboratoires publics ou privés. 


\title{
A first principle model for multiphase slugging flow in vertical risers
}

\author{
Florent Di Meglio, Glenn-Ole Kaasa, Nicolas Petit
}

\begin{abstract}
In this paper, we propose a simple model to represent the slugging flow regime appearing in vertical risers. We consider a one dimensional two-phase flow composed of a liquid phase and a gaseous compressible phase. The presented model can be applied to a wide class of systems, ranging from pure vertical risers to more complex geometries such as those found on actual sub sea petroleum facilities. Following ideas from the literature, we introduce a virtual valve located at the bottom of the riser. This allows us to reproduce observed periodic regimes. It also brings insight into the physics of the slugging phenomenon. Most importantly, this model reveals relatively easy to tune and seems suitable for control design. A tuning methodology is proposed along with a proof of the existence of a limit cycle under simplifying assumptions.
\end{abstract}

\section{INTRODUCTION}

In this paper, we study flows in risers, which are long pipes connecting reservoirs to surface facilities for oil production (see Figure 1). Severe slugging is a flow regime that arises mostly when entering tail production of an oil field. It is characterized by an unstable multiphase flow, where "slugs" of liquid accumulate before being pushed upwards by the gas. It is also characterized by oscillations of the pressure in the pipeline and oscillations of flow rates of gas and oil at the production end of the pipe. Although the phenomenon itself can be observed and sometimes reproduced on test rigs, its causes are not always known. The severe slugging flow regime can damage the installations and, most importantly, reduce the oil production. For these reasons, various techniques have been investigated in view of suppressing it.

A common setup where a riser is used is depicted in Figure 1. It consists of a wellhead (source), a surface separator, and a production choke at the outlet of the riser. The riser length typically ranges from a few hundred meters to several kilometers. To avoid instability, the most straightforward technique consists in choking down manually the pipes thanks to the choke located upstream the separator. Although this solution stabilizes the flow, it reduces significantly the oil production which, in turn, motivates the investigation of dynamic control of the valve. Indeed, it is possible, in closedloop, to stabilize at higher flow rates. PID controllers can be designed, using the bottom-hole pressure measurements as inputs for the feedback loop [1], [2], [3], but often, they require frequent re-tuning, and sometimes do not achieve stabilization at all. This yields a potential for model-based

F. Di Meglio (corresponding author) is a $\mathrm{PhD}$ candidate in Mathematics and Control at Mines Paristech, 60, Bd St-Michel, 75272 Paris, Cedex 06, France

G.O. Kaasa is Research Engineer at StatoilHydro ASA, Research Center Porsgrunn, Heroya Forksningspark 3908 Porsgrunn, Norway

N. Petit is Professor at Mines Paristech, 60, Bd St-Michel, 75272 Paris, Cedex 06, France control techniques and, consequently, motivates the elaboration of models able to reproduce severe slugging.

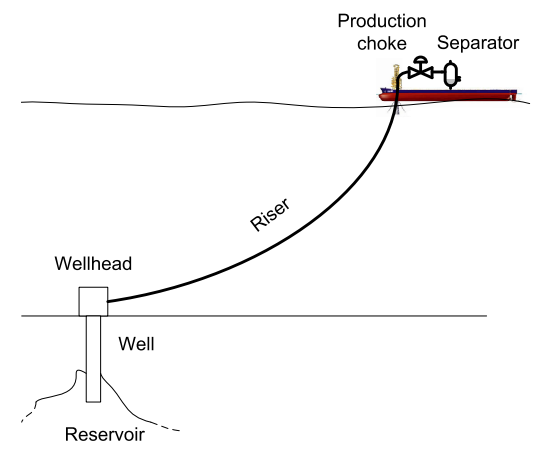

Fig. 1. Common riser setup

Two classes of models can be found in the literature. The most accurate type is based on (usually nonlinear) Partial Differential Equations (PDE) representing two-phase (oil and gas) or three-phase (oil, water and gas) flows. These models ([4], [5],s [6], [7], [8], [9]) are able to reproduce the slugging phenomenon in many cases, but fail to match the behavior of real-life wells in other cases, in particular when the instability comes from reservoir dynamics, which there is little knowledge about. Unfortunately, it is not possible to derive control laws from these models because of their complexity. The second class of models is based on Ordinary Differential Equations (ODE) and represents a different trade-off between accuracy and complexity. A prime example is the model presented in [10], which, besides its numerous merits, does not sufficiently rely on physics to accurately reproduce the physical response of the system. Besides, the model presented in [11] is too complicated for model-based control, and is difficult to tune. Finally, the model is not general enough and is designed for a specific geometry. This is also the case for the model of gas-lift presented in [12]. In this context, we believe that a key for control design of riser systems is the derivation of simple physics-inspired models capturing enough parameters to reproduce observed phenomena (while limiting the calibration effort). This paper represents a step toward this goal.

Our contribution is as follows. Consider a vertical riser subjected to a constant input flow. The output flow of the riser is controlled by a choke. Unstable flow regime can occur, especially when the choke is largely opened, which, unfortunately, corresponds to a point of industrial interest. This kind of instability is also observed on related systems: oil wells with a gas reservoir [13], [14], risers with low-point [11], [15], [16]. Generally, switches of valves are reported 
FrC08.1

to be at the birth of the oscillating phenomenas: downhole choke plays a key role in the "casing-heading" in [12], [17], while the geometric low-point acting as a valve is studied in [11]. In the riser considered here, no such valve exists or is even suggested by the geometry. Yet, we propose to model the riser using a "virtual choke" located at a wellchosen point at the bottom of the riser. In this approach, the riser is modeled as a three-state set of ordinary differential equations similar to those found in [12], [11], [18], [19]. We can tune the model analytically to fit most physical systems of interest.

The paper is organized as follows. After having briefly presented the physical system under consideration and the proposed model in Section II, we identify the successive stages of the slugging phenomenon. It appears in great accordance with real-world observed slugging systems. Then, based on this model, we investigate the existence of a limit cycle in Section III. In Section IV, we present a tuning methodology to calibrate the model and match the characteristics of the oscillations, such as the bifurcation point, frequency of oscillations and steady-state conditions. A couple of case studies are presented. Finally, in Section V, we discuss some of the limitations of the model and propose possible ways of improvement. Conclusions are given in Section VI.

\section{SYSTEM DESCRIPTION AND PROPOSED MODEL}

Consider the vertical riser depicted in Figure 1. To get insight into the slugging phenomenon, which can be observed even with constant inflows, we propose a model, pictured in Figure 2, based on first principles (mass balances). It is considered that the riser contains three distinct volumes, two of which are filled with gas, and separated by a horizontal virtual valve, while the last volume is filled with liquid. This scheme stresses the possible existence of an obstructing liquid interface at a certain location in the riser, which is modeled by the virtual valve, preventing the gas from flowing normally. This yields the formation of a large elongated bubble (pictured in Figure 2) behind the interface. In this elongated bubble, a pressure build-up occurs and eventually generate instabilities. We now detail the model. A complete nomenclature is given in Table I.

\section{A. Modelling assumptions}

1) Mass balances: The state variables are the masses of gas in the elongated bubble $\left(m_{g, e b}\right)$ and in the riser $\left(m_{g, r}\right)$, and the mass of liquid $\left(m_{l, r}\right)$ in the riser. Mass conservation yields

$$
\begin{aligned}
\dot{m}_{g, e b}(t) & =(1-\epsilon) w_{g, \text { in }}-w_{g}(t) \\
\dot{m}_{g, r}(t) & =\epsilon w_{g, \text { in }}+w_{g}(t)-w_{g, \text { out }}(t) \\
\dot{m}_{l, r}(t) & =w_{l, \text { in }}-w_{l, \text { out }}(t)
\end{aligned}
$$

where $w_{g, \text { in }}$ and $w_{g, \text { out }}$ (resp. $w_{l, \text { in }}$ and $w_{l, o u t}$ ) are the mass flow rates of gas (resp. liquid) entering (in) the riser and coming out (out) of the riser ; and $w_{g}$ is the mass flow rate of gas through the virtual downhole choke. Note that,

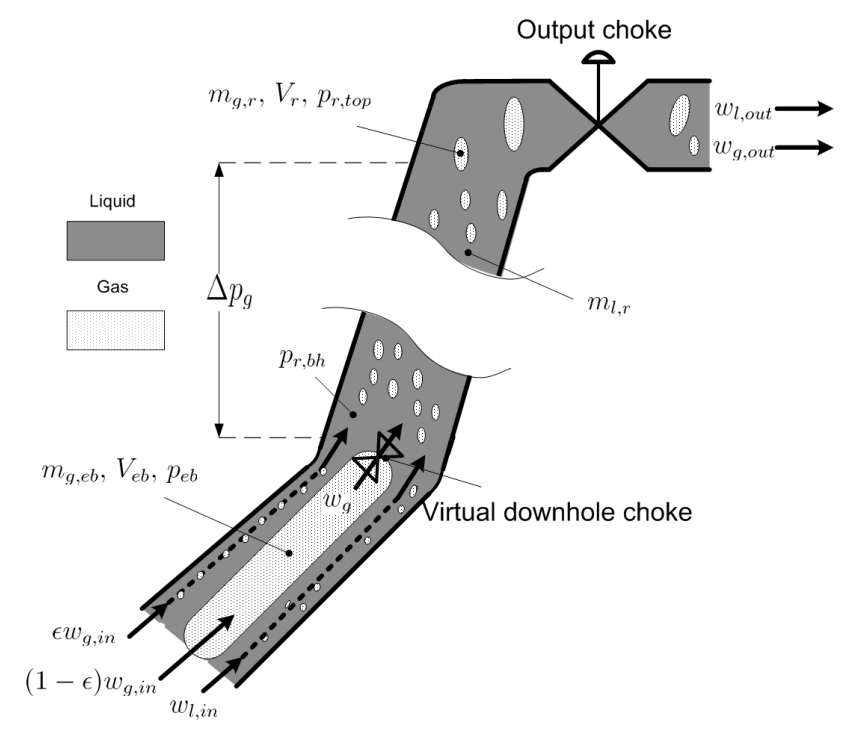

Fig. 2. Schematic diagram of the system

in this model, a fraction of the gas flow (determined by $\epsilon \in(0,1))$ goes directly in the upper part of the riser, whereas the remaining accumulates in the bottom part of volume $V_{e b}$, causing a build-up of pressure. Besides, we make the following assumptions, which will be discussed further in Section V.

2) Description of mass flows: As mentioned above, the inflows of gas $\left(w_{g, i n}\right)$ and liquid $\left(w_{l, i n}\right)$ are assumed constant. The mass of gas in the riser is negligible compared to the mass of liquid in the riser. Therefore, if $w_{\text {out }}$ is the total mass flow rate through the production choke, one has

$$
\begin{aligned}
& w_{g, \text { out }}=\frac{m_{g, r}}{m_{g, r}+m_{l, r}} w_{\text {out }} \approx \frac{m_{g, r}}{m_{l, r}} w_{\text {out }} \\
& w_{l, \text { out }}=\frac{m_{l, r}}{m_{g, r}+m_{l, r}} w_{\text {out }} \approx w_{\text {out }}
\end{aligned}
$$

Besides, all the non constant flows are given by linearized valve equations: they are linear functions of the pressure drop over the choke under consideration (actual or virtual). We also assume that there is no flowback through these valves. This yields the following expressions

$$
\begin{aligned}
w_{g} & =C_{g} \max \left(p_{e b}-p_{r, b h}, 0\right) \\
w_{\text {out }} & =C_{c} \max \left(p_{r, t o p}-p_{s}, 0\right) u
\end{aligned}
$$

where $p_{e b}$ is the pressure of the gas in the elongated bubble, $p_{r, b h}$ is the pressure of gas downstream this choke (bottom hole), $p_{r, t o p}$ is the pressure upstream the production choke and $p_{s}$ is the separator pressure. $C_{g}$ and $C_{c}$ are positive constants.

3) Determination of pressures: The pressures in the riser are given by the ideal gas law. The volume of the elongated bubble $V_{e b}$ is assumed to be constant, whereas the gas downstream the virtual choke is assumed to be compressible. Its volume $V_{g, r}$ depends on the mass of liquid in this part: $V_{g, r}=V_{r}-\frac{m_{l, r}}{\rho_{l}}$ where $V_{r}$ is the volume of the riser. Besides, 
the pressure drop over the riser is supposed to be gravitydominated (friction being neglected)

$$
\begin{aligned}
p_{e b} & =\frac{R T}{M V_{e b}} m_{g, e b} \\
p_{r, b h} & =p_{r, t o p}+\frac{g \sin \theta}{A} m_{l, r} \\
p_{r, t o p} & =\frac{R T}{M\left(V_{r}-\frac{m_{l, r}}{\rho_{l}}\right)} m_{g, r}
\end{aligned}
$$

where $\theta$ is the mean inclination of the pipe, and $A$ the crosssection area.

\section{B. Sustained oscillations of the proposed model}

The proposed model (1)-(2)-(3) can reproduce oscillations observed in experimental data such as that obtained from a multiphase flow rig at StatoilHydro's research center in Porsgrunn, and also results obtained with state-of-the-art multiphasic flow simulator OLGA (c). Figure 3 shows the oscillations of pressures and mass flow rates of the model compared to those of the OLGA model. The system under consideration, which corresponds to the experimental rig of Porsgrunn, is a $80 \mathrm{~m}$-long pipe, composed of a nearly horizontal part of $70 \mathrm{~m}$ followed by a vertical part of $10 \mathrm{~m}$. The first part of the riser is slightly inclined downwards, therefore the bottom of the vertical part is the lowest point of the system. Following an idea from [11], this low-point is where we assume that the virtual valve is located at. Under this assumption, the volume $V_{1}$ corresponding to the volume of the elongated bubble is found to fill almost entirely the horizontal part of the riser. To obtain the relatively accurate

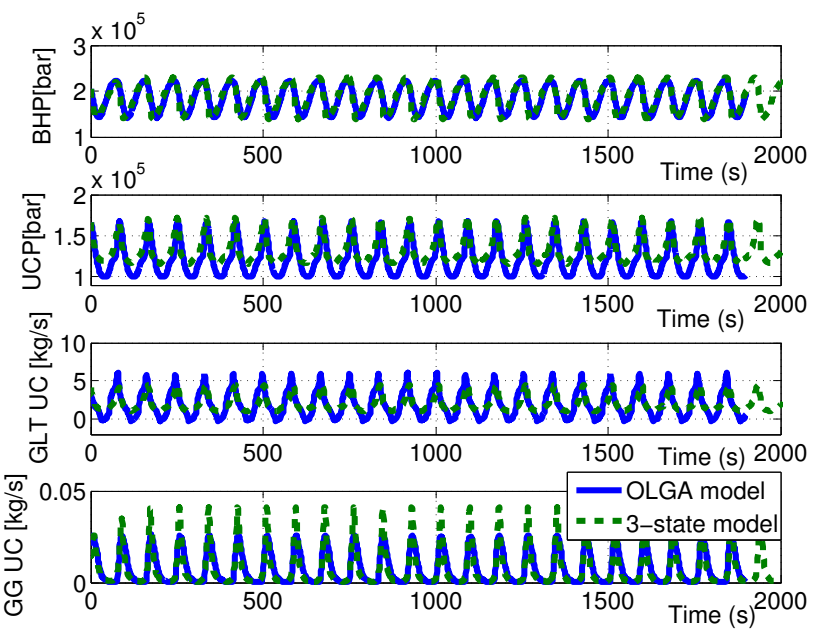

Fig. 3. Pressure and mass flow rate oscillations: model vs OLGA

fit of the model to the OLGA data in Figure 3, the proposed model needs to be tuned. A tuning methodology is detailed in Section IV. Before focusing on the theoretical existence of a limit cycle for the proposed model, we now qualitatively analyze the oscillations of the system. Three phases, depicted in Figure 4 can be identified. The oscillations are created by the accumulation of gas in volume $V_{e b}$, which can only be emptied through the virtual valve if the pressure $p_{e b}$ is greater

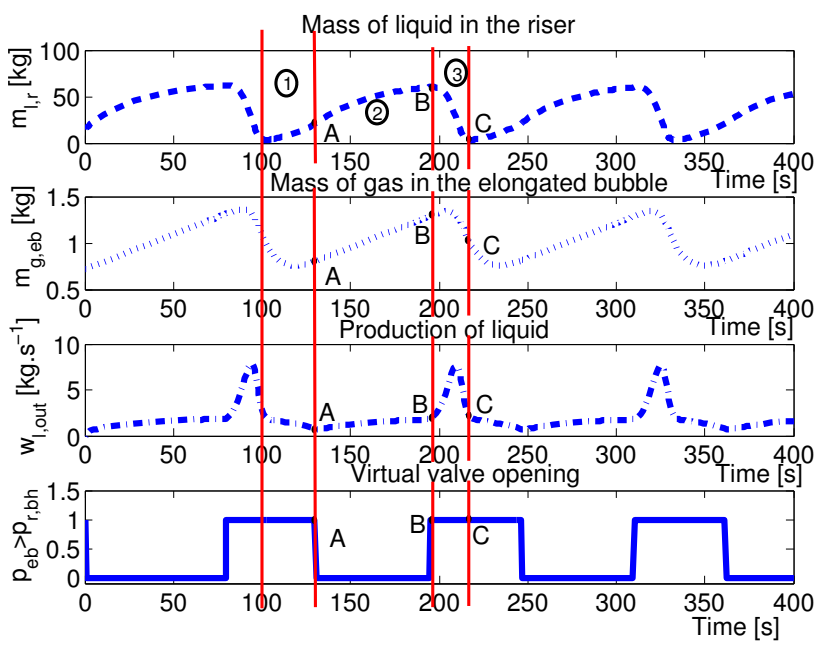

Fig. 4. The three stages of the oscillations

than the bottom-hole pressure $p_{r, b h}$. The three phases are as follows.

a) Phase 1: The bottom hole pressure is low because the mass of liquid in the riser is small and causes only a small gravity pressure drop. Therefore, the virtual valve is open $\left(p_{r, b h}<p_{e b}\right)$ and gas flows out from $V_{e b}$ at a high rate. Besides, the output flows are low, so that the mass of liquid and $p_{r, b h}$ increase due to the constant input flows. When $p_{r, b h}$ gets large enough, the flow through the virtual valve decreases, and $V_{e b}$ starts filling again. Yet, $m_{l, r}$ increases more rapidly than $m_{g, e b}$, and therefore the valve closes $\left(p_{r, b h} \geq p_{e b}\right)$ at point $\mathrm{A}$ in the timeline.

b) Phase 2: After a sharp increase, $m_{l, r}$ reaches an asymptotic value, whereas $m_{g, e b}$ increases steadily. Therefore, the virtual valve remains closed until $p_{e b}$ reaches the asymptotic value of $p_{r, b h}$ and the valve opens again at point $\mathrm{B}$ in the timeline.

c) Phase 3: When the valve opens, the riser is filled with liquid (the value of $m_{l, r}$ is high) and the gas entering the upper part of the riser is highly compressed. This increases the pressure at the choke and therefore the outflows get very high: the riser is suddenly emptied of its liquid and gas. It is the blow-out phase. After this blow-out, the masses, the flows and the pressures go back to low values, and, eventually, the cycle repeats when point $\mathrm{C}$ in the timeline is reached.

We now focus on a more quantitative approach by investigating the existence of a periodic orbit.

\section{EXISTENCE OF A LIMIT CYCLE UNDER SIMPLIFYING ASSUMPTIONS}

\section{A. Motivations and simplifications}

Classically, existence of a limit cycle is analyzed for planar dynamical systems using the Poincaré-Bendixson criterion [20]. As a matter of fact, one can easily realize that along the observed limit cycle, the three dimensional state-space model (1)-(2)-(3) can be reduced to a two-dimensional state model. When $w_{g}=0$, there exists an affine relation between $m_{g, r}$ and $m_{l, r}$. This point is illustrated in Figure 5 where the 


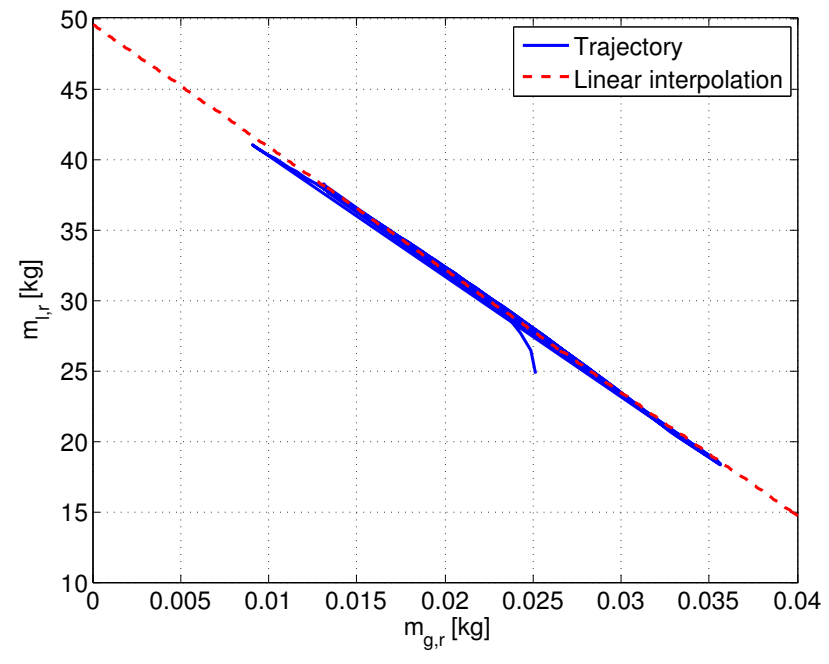

Fig. 5. Mass of gas in the riser versus mass of liquid when the virtual choke is closed

relevance of a model reduction through a linear fit of $m_{l, r}$ in terms of $m_{g, r}$ is stressed.

Although this relation only holds perfectly during the phases where the downhole choke is closed, it is expected that it will not affect the slugging behavior to assume that some linear relation holds for all times. We now investigate the validity this statement.

The coefficient for the linear fit are chosen so that they respect the steady-state equations. Thus, we seek two coefficients $k_{1}$ and $k_{2}$, consistent with the equilibrium values, and such that $m_{l, r}=k_{1} m_{g, r}+k_{2}$. This yields $k_{1}=-\frac{b}{p_{s}+\frac{w_{l, i n}}{u C_{c}}}$ and $k_{2}=$ $m_{l, r}^{*}$. As a consequence, the upstream choke pressure remains constant, equal to its equilibrium value $p_{c, e q}=p_{s}+\frac{w_{l, i n}}{u C_{c}}$. Despite these simplifications, the model still reproduces the oscillations of the bottom hole pressure, which shows that the slugging structure of the model has been preserved throughout these changes. These considerations yield the following simplified model, where the variables $m_{g, e b}$ and $m_{g, r}$ have been rewritten $x_{1}$ and $x_{2}$

$$
\begin{aligned}
& \dot{x_{1}}=(1-\epsilon) w_{g, \text { in }}-\max \left(\alpha x_{1}-\gamma+\beta x_{2}, 0\right) \\
& \dot{x_{2}}=\epsilon w_{g, \text { in }}+\max \left(\alpha x_{1}-\gamma+\beta x_{2}, 0\right)-\delta \frac{x_{2}}{c-x_{2}}
\end{aligned}
$$

where the constants $\alpha, \beta, \gamma, \delta w_{g, \text { in }}$ and $c$ are expressed in terms of the model variables and the fit coefficients $k_{1}$ and $k_{2}$ in Table I, and were introduced for clarity purposes. We now perform an analysis of the reduced dynamical system (4)-(5).

\section{B. Notations and assumptions}

Consider the compact rectangular domain $D \stackrel{\text { def }}{=}\left[0, x_{1}^{*}\right] \times\left[0, x_{2}^{*}\right]$ where

$$
\begin{aligned}
x_{1}^{*} & =\frac{\gamma+\beta c-\delta-\epsilon w_{g, \text { in }}}{\alpha} \\
x_{2}^{*} & =c \sqrt{1-\frac{\delta}{2 \beta c}}
\end{aligned}
$$

Its boundary is $\partial D \stackrel{\text { def }}{=} D_{1} \cup D_{2} \cup D_{3} \cup D_{4}$. We also define the four unit vectors $V_{i}, i \in\{1,2,3,4\}$ orthogonal to the four components of $\partial D$ and pointing outside of $D$. The expressions of all these parameters are given in Table I. Eventually, we make the following assumption

$$
\beta c>\delta+w_{g, \text { in }}
$$

so that $x_{1}^{*}$ and $x_{2}^{*}$ are properly defined and strictly positive.

\section{Main result}

Proposition 3.1: Under the assumption $-\alpha+\beta-$ $\frac{1}{c} \frac{\left(w_{g, i n}+\delta\right)^{2}}{\delta}>0$, system (4)-(5) has a periodic orbit lying in $D$. Conversely, if $-\alpha+\beta-\frac{1}{c} \frac{\left(w_{g, i n}+\delta\right)^{2}}{\delta}<0$, system (4)-(5) has a unique (locally) asymptotically stable stationary point.

1) Preliminary lemma: The proof of the proposition relies on the following lemma.

Lemma 3.2: Every trajectory of (4)-(5) starting in $D$ remains in $D$ for all future times.

Proof: We prove that for all $x \in \partial D$, the right-hand side

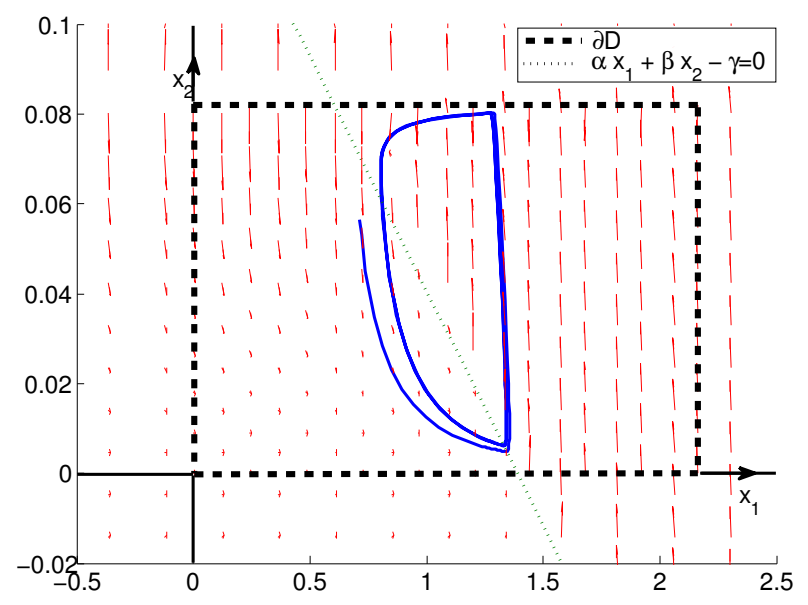

Fig. 6. The trajectories of the system remain in D

of the differential equations (4)-(5), $f(x) \stackrel{\text { def }}{=}\left(f_{1}(x), f_{2}(x)\right)$, points inside of $D$, as shown in Figure 6. Indeed, one has

$$
\left.\forall x \in D_{1}, \quad f_{2}(x)=\epsilon w_{(} g, i n\right)+\max \left(\alpha x_{1}-\gamma, 0\right)>0
$$

so that $\forall x \in D_{1}, f(x) \cdot V_{1}<0$. One should notice that, this calculus being valid for any $x_{1}$, if $x_{2}$ is strictly positive at initial time, it remains so for all future times. Besides,

$$
\forall x \in D_{4}, \quad f_{1}(x)=(1-\epsilon) w_{g, \text { in }}-\max \left(-\gamma+\beta x_{2}, 0\right)>0
$$

so that $\forall x \in D_{4}, f(x) \cdot V_{4}<0$.

Similarly to $x_{2}$, if $x_{1}>0$ at initial time, then $x_{1}>0$ for all future times. On $D_{2}$, one can first realize that assumption (A1) yields $\alpha x_{1}^{*}+\beta x_{2}-\gamma>0$. Therefore,

$$
\forall x \in D_{2}, \quad f_{1}(x)=-\left(\beta c-\delta-w_{g, \text { in }}+\beta x_{2}\right)
$$

According to assumption (A1), $\beta c-\delta-w_{g, \text { in }}>0$, and we have shown that $x_{2}$ is positive, therefore, $\forall x \in D_{2}, f(x) \cdot V_{2}<0$.

Once again, this result is valid for any $x_{2}$, so that $x_{1}<x_{1}^{*}$ for all times. On $D_{3}$, further investigation is needed. We aim 
at proving that $f_{2}(x)=\epsilon w_{g, \text { in }}+\max \left(\alpha x_{1}-\gamma+\beta x_{2}^{*}, 0\right)-\delta \frac{x_{2}^{*}}{c-x_{2}^{*}}$ is strictly negative for all $x_{1}<x_{1}^{*}$. It is sufficient to prove the result when $\alpha x_{1}-\gamma+\beta x_{2}^{*}>0$, for the result will hold when it is not the case thanks to the max function. Using the expression of $x_{2}^{*}$ and the fact that $x_{1}<x_{1}^{*}$, one can easily prove that

$$
\forall x \in D_{3}, f_{2}(x)<-\frac{\delta}{2} \frac{1}{1-\sqrt{1-\frac{\delta}{2 \beta c}}}<0
$$

Therefore $\forall x \in D_{3}, f(x) \cdot V_{3}<0$, which achieves the proof of the lemma.

2) Proof of the main result: Using this lemma, we now prove Proposition 3.1.

Proof: Solving the equilibrium equations, one easily sees that the system can only have one equilibrium, defined by

$$
\bar{x}_{1}=\frac{(1-\epsilon) w_{g, i n}+\gamma-\beta \frac{w_{g, i n}}{w_{g, i n}+\delta} c}{\alpha}, \quad \bar{x}_{2}=\frac{w_{g, i n}}{w_{g, i n}+\delta} c
$$

The Jacobian matrix at this point has the following expression

$$
J=\left(\begin{array}{cc}
-\alpha & -\beta \\
\alpha & \beta-\frac{1}{c} \frac{\left(w_{g, i n}+\delta\right)^{2}}{\delta}
\end{array}\right)
$$

and its determinant is $\operatorname{det}(J)=\frac{\alpha}{c} \frac{\left(w_{g, i n}+\delta\right)^{2}}{\delta}>0$. Therefore, the equilibrium point is asymptotically stable for the linearized system if

$$
\operatorname{tr}(J)=-\alpha+\beta-\frac{1}{c} \frac{\left(w_{g, i n}+\delta\right)^{2}}{\delta}<0
$$

and it is unstable if $\operatorname{tr}(J)>0$. If the equilibrium point is unstable, the Poincaré-Bendixson theorem can be applied thanks to Lemma 3.2. This shows the existence of a periodic orbit lying in $D$ for the nonlinear system (4)-(5). If $\operatorname{tr}(J)<0$, the equilibrium point is also asymptotically stable for the nonlinear system (see e.g. [20], Theorem 4.7). Interestingly, a conservative estimation of the basin of attraction can be obtained from a Lyapunov analysis. It consists of finding a positively invariant set $\Gamma$ lying in $D$, containing the origin, on which the following Lyapunov function

$$
V\left(\tilde{x}_{1}, \tilde{x}_{2}\right)=\frac{1}{2}\left(\frac{\rho\left(\tilde{x}_{2}\right)}{\alpha}{\tilde{x_{2}}}^{2}+\left(\tilde{x}_{1}+\tilde{x}_{2}\right)^{2}\right)
$$

is decreasing. This construction is detailed in appendix A.

\section{TUNING OF THE MODEL}

Furthering the previous qualitative analysis, it is possible to quantitatively fit measured oscillations. In details, the model (1)-(2)-(3) can be tuned to meet the following requirements

1) its equilibrium can correspond to desired pressures and mass flows,

2) its bifurcation point (i.e. occurrence of instability) can match the critical production choke opening value,
3) finally, the period of oscillations can match that of observed ones.

In the following, an analytic study of the model leads to a "plug-and-play" tuning procedure, requiring solely very limited human input.

\section{A. Matching steady-state operations}

a) Equation of equilibrium: There can only be an equilibrium if the two max functions appearing in (1) and (2) have strictly positive values, because the input flow rates are both non zero. In this case, we have

$$
p_{r, t o p, e q}=p_{s}+\frac{w_{l, i n}}{u C_{c}}
$$

which yields $m_{l, e q}=\frac{p_{r, t o p, e q}}{p_{r, t o p, e q}+b G L R} m_{l, r}^{*}$, where $b=\frac{\rho_{l} R T}{M}$ and $G L R=\frac{w_{g, i n}}{w_{l, i n}}$. Eventually, this yields the following expression of the bottom hole pressure

$$
p_{r, b h, e q}=p_{s}+\frac{w_{l, i n}}{C_{c} u}+\frac{m_{l, r}^{*} g \sin \theta}{A} \frac{p_{s}+\frac{w_{l, i n}}{C_{c} u}}{p_{s}+\frac{w_{l, i n}}{C_{c} u}+\frac{\rho_{l} R T}{M} \frac{w_{g, i n}}{w_{l, i n}}}
$$

b) Tuning of the steady-state point: The steady-state values of the upstream choke and bottom hole pressure are the most important values to be fitted, because they are the two variables that are usually measured with the best accuracy. They both depend on the value of the production choke opening $u$, which is why one should try to tune the "equilibrium curve", i.e. the values of the bottom hole or production choke pressure for a set of values of $u$.

The upstream choke pressure at the equilibrium is given by (8). This relation is affine in $\frac{1}{u}$, whereas it is traditionally affine in $\frac{1}{u^{2}}$ in the literature (see Section V-A). For this reason, we can only try to approximate the steady-state behavior away from $u=0$. Figure 7 shows the comparison between our steady-state model and the one obtained with the reference model from OLGA.

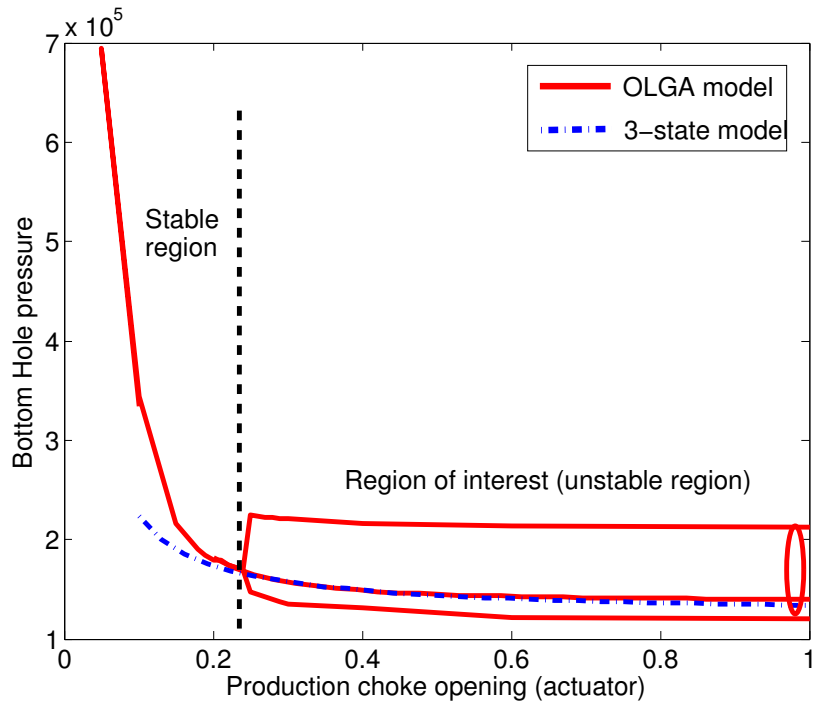

Fig. 7. Steady-state values of the bottom hole pressure with respect to choke opening 
Besides, $p_{s}$ being known with a good accuracy, one should choose either $w_{l, \text { in }}$ or $C_{c}$ to tune this value and make it match the given data. One should notice that $w_{l, i n}$ is the steady-state value of the outflow of liquid through the production choke, and that it is known up to the accuracy of the measurements of that flow (its range is usually known). One should also notice that, given the expression of $p_{r, b h, e q}$, once $p_{c, e q}$ has been chosen, there is no additional parameter available to tune the equilibrium value of the bottom hole pressure. This issue is discussed in Section V-C.

\section{B. Occurrence of instability}

In order to study the stability of the system around the equilibrium point, one can compute the Jacobian matrix and its eigenvalues. As is shown in Section III for the reduced model, the system oscillates when one of these eigenvalues is located in the right-half plane, and is stable otherwise. Unfortunately, this fact cannot be shown rigorously for the complete three-dimensional system as there does not exist any equivalent to the Poincaré-Bendixson theorem. Yet, the behavior (as observed on simulations) of the complete system is the same: when the equilibrium point is stable, it is locally attractive, conversely the system reaches a limit cycle when the equilibrium is unstable.

1) Matching (choke opening) bifurcation point: When increasing the opening of the production choke, we observe that the system switches from a stable behavior to a slugging behavior. This can also be seen by plotting the eigenvalues of the system against the choke opening, and noticing that they cross the imaginary axis for a certain value of the choke opening as shown in Figure 8.

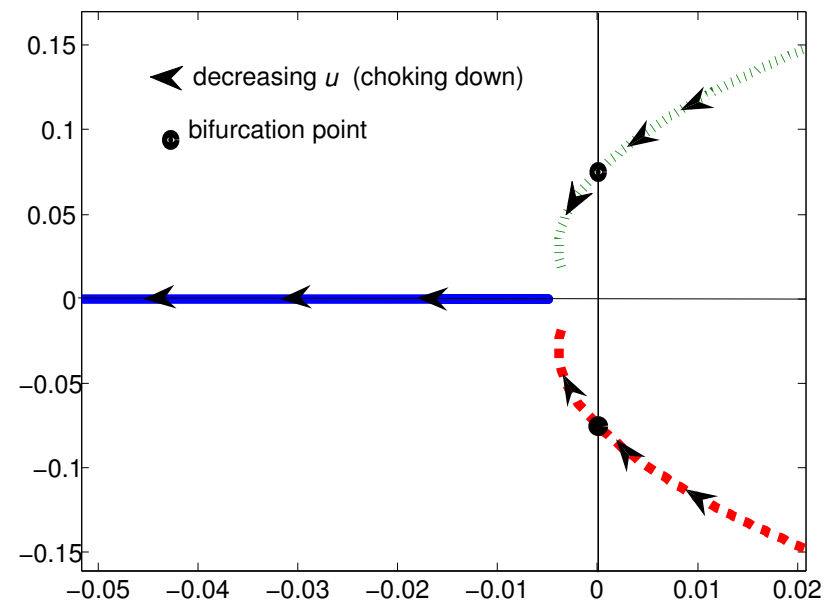

Fig. 8. Locus of the eigenvalues when decreasing $u$

This phenomenon is well-known by the petroleum engineers, who observed that the slugging could be suppressed by choking down the riser, which, unfortunately, also reduces the production rate. Yet, the value of the choke opening for which the system stops slugging, i.e. the bifurcation point, can be tuned.

To correctly fit the value of that point, one should write the Routh stability criterion and solve it for the parameter to tune so that the bifurcation point of the model and that of the real system coincide. Unfortunately, the criterion does not take the form of a tractable expression, and the bifurcation point has to be set through iterative numerical methods, by picking one parameter to tune and finding numerically its optimal value (i.e. the one that gives the best value of $u$ at which instability occurs).

2) Matching frequency of oscillations: The frequency of the oscillations at the bifurcation point is given by the imaginary part of the eigenvalues of the Jacobian matrix, because the oscillations are purely linear at that point. In details, one can numerically tune a single parameter to act on the imaginary part of the eigenvalues and tune the frequency. This only allows us to tune the frequency for a given choke opening, and the frequency may differ for other values of $u$.

\section{LIMITATIONS OF THE MODEL AND POSSIBLE UPGRADES}

To maximize the model ability to reproduce the behavior of real fields, several enhancements (by means of model refinements) can be considered. Each of them has a cost which is now briefly discussed.

\section{A. Nonlinear valve equations}

The assumption that the outflow $w_{\text {out }}$ is linear with respect to the pressure drop over the choke is debatable. Indeed, the valve equations are traditionnaly of the form

$$
w_{\text {out }}=C \sqrt{\rho_{\text {mix }} \Delta p}
$$

where $\rho_{\text {mix }}$ is the mixture density. This form is known to be appropriate in the cases of monophasic flow, but no such general formula can be found in the literature for multiphasic flow. The assumption of a linear relation was made here to simplify the analysis. Still, it is possible to replace it with a more general expression without compromising the tuning possibilities, for example by using a relation of the form

$$
w_{\text {out }}=C(\rho \Delta p)^{1 / n}
$$

with $n \geq 1$. Even though such equation increases the generality to the model, it also requires a manual tuning on $n$ which we would like to avoid. Yet, it reveals handy in some cases, especially when studying field data.

\section{B. Non constant inflows}

In the current model, the inflows $w_{l, \text { in }}$ and $w_{g, \text { in }}$ are assumed constant. We know that this is not the case in real fields, but the assumption is considered for two reasons. First, the model, contrary to many others, does not need a reservoir model to reproduce slugging. Secondly, there is, up to our knowledge, no simple model of reservoir that gives good results compared to real-world data.

The main effect of considering constant inflows is that the steady-state values of the outflows remains unchanged when the system slugs or not. This is inconsistent with reality, where the outflows are lower in slugging mode. Therefore, one can investigate the addition of a productivity index (PI) type of model reservoir. This will surely complicate the analysis (in particular the computation of the steady-state) and therefore reduce the tuning possibilities. 


\section{Steady-state bottom hole pressure}

As is, the steady-state bottom hole pressure is given by

$$
p_{r, b h, e q}=p_{s}+\frac{w_{l, i n}}{C_{c} u}+\frac{m_{l, r}^{*} g \sin \theta}{A} \frac{p_{s}+\frac{w_{l, i n}}{C_{c} u}}{p_{s}+\frac{w_{l, i n}}{C_{c} u}+\frac{\rho_{l} R T}{M} \frac{w_{g, i n}}{w_{l, i n}}}
$$

The $C_{c}$ parameter is already used to tune the upstream choke pressure, and the range of the $w_{l, \text { in }}$ and $w_{g \text {,in }}$ parameters is given by measurements. The other parameters being fixed, this means we have very few margin to tune the value of the bottom hole pressure. This problem turns out to be important when studying real field data, where the gravity pressure drop in the riser plays an important role.

To address this issue, it is possible to assume that there is a still mass of liquid that remains constant but impacts on the system near the bottom hole and increases the pressure there. This point is currently under investigation.

\section{Conclusion}

We have presented a model for slugging flow in risers, which can be applied to a wide class of geometries, including purely vertical risers or systems with a low-point. We have shown that this system could be tuned to reproduce the behavior of an experimental rig with a good accuracy. The improvements of Section V seem necessary to reproduce the oscillation of real wells, while our preliminary simulations are promising. We will now aim at designing an observer for this model. Two directions are under investigation: the design of a nonlinear observer in the classic sense, for example with a Lyapunov analysis based on the proposed reduced model, and the design of a frequency tracking and phase locking system on field measurements. The long-term goal is, of course, to design a control law that would stabilize the system at high production choke opening values, but the quality of the model and its ability to fit real-scale risers has first to be more precisely assessed.

\section{REFERENCES}

[1] E. F. Blick, P. Enga, and P.-C. Lin, "Stability analysis of flowing oil wells and gas-lift wells," SPE Production Engineering, vol. 3, No 4, pp. 508-514, 1986.

[2] G. O. Eikrem, B. Foss, L. Imsland, B. Hu, and M. Golan, "Stabilization of gas lifted wells," Proceedings of the 15th IFAC World Congress, vol. 15, Part 1, 2002.

[3] M. Dalsmo, E. Halvorsen, and O. Slupphaug, "Active feedback control of unstable wells at the brage field," SPE Annual Technical Conference, 2002.

[4] Scandpower, OLGA (C2000 User's Manual. Scandpower, 2004.

[5] D. Ferre, V. Bouvier, and C. Pauchon, TACITE Physical Model Description Manual. Rapport IFP, 1995.

[6] A. E. Dukler and M. G. Hubbard, "A model for gas-liquid slug flow in horizontal and near horizontal tubes," Industrial $\mathcal{E}$ Engineering Chemistry Fundamentals, vol. 14, no. 4, pp. 337-347, 1975.

[7] Y. Taitel, "Stability of severe slugging," International Journal of Multiphase Flow, vol. 12, no. 2, pp. 203 - 217, 1986.

[8] Y. Taitel and D. Barnea, "Two-phase slug flow," Advances in Heat Transfer, vol. 20, pp. 83 - 132, 1990.

[9] A. M. Ansari, U. Sylvester, O. Shoham, and U. Brill, "A comprehensive mechanistic model for upward two-phase flow in wellbores." $S P E$ Annual Technical Conference, 1990.

[10] G.-O. Kaasa, "Attenuation of slugging in unstable oil by nonlinear control," IFAC, 2008.

\begin{tabular}{|c|c|c|}
\hline Variable & Description & Unit \\
\hline$w_{g, \text { in }}$ & $\begin{array}{c}\text { Mass flow rate of gas } \\
\text { entering the riser }\end{array}$ & $k g \cdot s^{-1}$ \\
\hline$w_{l, i n}$ & $\begin{array}{c}\text { Mass flow rate of liquid } \\
\text { entering the riser }\end{array}$ & $k g . s^{-1}$ \\
\hline$w_{g}$ & $\begin{array}{l}\text { Mass flow rate of gas } \\
\text { through the virtual choke }\end{array}$ & $k g . s^{-1}$ \\
\hline$w_{g, \text { out }}$ & $\begin{array}{l}\text { Mass flow rate of gas } \\
\text { coming out of the riser }\end{array}$ & $k g \cdot s^{-1}$ \\
\hline$w_{l, o u t}$ & $\begin{array}{l}\text { Mass flow rate of liquid } \\
\text { coming out of the riser }\end{array}$ & $k g . s^{-1}$ \\
\hline$m_{g, e b}$ & $\begin{array}{l}\text { Mass of gas in } \\
\text { the elongated bubble }\end{array}$ & $\mathrm{kg}$ \\
\hline$m_{g, r}$ & Mass of gas in the riser & $k g$ \\
\hline$m_{l, r}$ & Mass of liquid in the riser & $k g$ \\
\hline$p_{e b}$ & Pressure in the elongated bubble & $\mathrm{Pa}$ \\
\hline$p_{r, b h}$ & Bottom hole pressure in the riser & $\mathrm{Pa}$ \\
\hline$p_{r, t o p}$ & Pressure at the top of the riser & $\mathrm{Pa}$ \\
\hline$p_{s}$ & Separator pressure & $\mathrm{Pa}$ \\
\hline$V_{e b}$ & Volume of the elongated bubble & $m^{3}$ \\
\hline$V_{r}$ & Volume of the riser & $m^{3}$ \\
\hline$A$ & Section of the pipe & $m^{2}$ \\
\hline$\theta$ & Mean inclination of the riser & $\mathrm{rad}$ \\
\hline$C_{c}$ & Production valve constant & $m^{-2} \cdot s$ \\
\hline$C_{g}$ & Virtual valve constant & $m^{-2} \cdot s$ \\
\hline$\rho_{l}$ & Liquid density & kg. $\mathrm{m}^{-3}$ \\
\hline$M$ & Molar mass of the gas & kg.mol ${ }^{-1}$ \\
\hline$T$ & Temperature in the riser & $K$ \\
\hline$R$ & Ideal gas constant & J.mol ${ }^{-1} \cdot K^{-1}$ \\
\hline$g$ & Standard gravity constant & $m \cdot s^{-2}$ \\
\hline$b$ & $\frac{\rho_{l} R T}{M}$ & $\mathrm{~Pa}$ \\
\hline$\alpha$ & $\frac{C_{g} R T}{M V_{e b}}$ & $s^{-1}$ \\
\hline$\beta$ & $-\frac{g k_{1} \sin \theta}{A} C_{g}$ & $s^{-1}$ \\
\hline$\gamma$ & $-\frac{b}{k_{1}} C_{g}+\frac{g k_{2} \sin \theta}{A} C_{g}$ & $k g . s^{-1}$ \\
\hline$\delta$ & $-\frac{w_{l, i n} p_{c, e q}}{b}$ & $k g \cdot s^{-1}$ \\
\hline$c$ & $-\frac{k_{2}}{k_{1}}=\frac{m_{l, r}^{*} p_{c, e q}}{b}$ & $k g$ \\
\hline
\end{tabular}

TABLE I

NOMENCLATURE

[11] E. Storkaas, "Control solutions to avoid slug flow in pipeline-riser systems," Ph.D. dissertation, Norwegian University of Science and Technology, 2005.

[12] L. Sinègre, "Dynamic study of unstable phenomena stepping in gaslift activated systems," Ph.D. dissertation, Ecole des Mines de Paris, 2006.

[13] A. J. Torre, R. N. Blais, J. Brill, D. Doty, and Z. Schmidt, "Casingheading in flowing wells," in SPE Production Operations Symposium, no. SPE 13801, 1987, oklahoma City, Oklahoma.

[14] E. Blick and L. Boone, "Stabilization of naturally flowing oil wells using feedback control," SPE Paper, 1986.

[15] V. Henriot, E. Duret, E. Heintz, and A. Courbot, "Multiphase production control: Application to slug flow," Oil $\mathcal{E}$ Gas Science and Technology - Rev IFP, vol. 57, No. 1, pp. 87-98, 2002.

[16] E. Duret, "Dynamique et contrôle des écoulements polyphasiques," Ph.D. dissertation, Ecole des Mines de Paris, 2005.

[17] B. Hu, "Characterizing gas-lift instablilities," Ph.D. dissertation, Department of Petroleum Engineering and Applied Geophysics, NTNU, 2004.

[18] O.-M. Aamo, G. Eikrem, H. Siahaan, and B. Foss, "Observer design for multiphase flow in vertical pipes with gas-lift theory and experiments," Journal of Process Control, vol. 15, pp. 247-257, 2005.

[19] B. Jansen, M. Daslmo, L. Nøkleberg, K. Havre, V. Kristiansen, and P. Lemetayer, "Automatic control of unstable gas lifted wells," SPE annual technical conference, 1999.

[20] H. K. Khalil, Nonlinear Systems, 3rd ed. Upper Saddle River, N.J.: Prentice Hall, 2002.

[21] A. Loría and E. Panteley, "Uniform exponential stability of linear time- 
varying systems: revisisted," Systems $\mathcal{E}$ control letters, vol. 47, No 1, pp. 13-24, 2002.

\section{APPENDIX}

\section{A. Basin of attraction}

As mentioned in III-C.2, the basin of attraction of the point of system (4)-(5) can be estimated. In the open set $D^{+} \subset D$ where the max functions are strictly positive, the system can be rewritten as

$$
\begin{aligned}
& \dot{\tilde{x}}_{1}=-\alpha \tilde{x}_{1}-\beta \tilde{x}_{2} \\
& \dot{\tilde{x}}_{2}=\alpha \tilde{x}_{1}+\beta \tilde{x}_{2}-\rho\left(\tilde{x}_{2}\right) \tilde{x}_{2}
\end{aligned}
$$

where $\tilde{x}_{1}=x_{1}-\overline{x_{1}}, \tilde{x}_{2}=x_{2}-\overline{x_{2}}$ and $\rho\left(\tilde{x}_{2}\right)=\frac{w_{g, i n}+\delta}{c-\bar{x}_{2}-\tilde{x}_{2}}$. This system will now be our system of interest. We denote $\tilde{D}^{+}$the set corresponding to $D^{+}$, centered around the equilibrium in the $\left(\tilde{x}_{2}, \tilde{X}\right)$ coordinates. Given the result of Lemma 3.2, we have, for any trajectory starting in $\tilde{D}^{+}$

$$
0<\frac{w_{g, \text { in }}+\delta}{c-x_{2}^{*}}=\rho_{\min }<\rho\left(\tilde{x}_{2}(t)\right)<\rho_{\max }=\frac{w_{g, \text { in }}+\delta}{c}
$$

Following the approach of [21], we put the system into the following form, by replacing $\tilde{x}_{1}$ with a new variable $\tilde{X}=$ $\tilde{x}_{1}+\tilde{x}_{2}$

$$
\left(\begin{array}{c}
\dot{\tilde{x}}_{2} \\
\dot{\tilde{X}}
\end{array}\right)=\left(\begin{array}{ll}
A\left(\tilde{x}_{2}\right) & \alpha \\
-\rho\left(\tilde{x}_{2}\right) & 0
\end{array}\right)\left(\begin{array}{c}
\tilde{x}_{2} \\
\tilde{X}
\end{array}\right)
$$

where $A\left(\tilde{x}_{2}\right)=-\alpha+\beta-\rho\left(\tilde{x}_{2}\right)$. Consider the candidate Lyapunov function (7), written in the $\left(\tilde{x}_{2}, \tilde{X}\right)$ coordinates $V\left(\tilde{x}_{2}, \tilde{X}\right)=\frac{1}{2}\left(\begin{array}{c}\tilde{x}_{2} \\ \tilde{X}\end{array}\right)^{T} R(t)\left(\begin{array}{c}\tilde{x}_{2} \\ \tilde{X}\end{array}\right)$ where $R\left(\tilde{x}_{2}, \tilde{X}\right)=$ $\left(\begin{array}{cc}P\left(\tilde{x}_{2}\right) & 0 \\ 0 & 1\end{array}\right)$ and $P\left(\tilde{x}_{2}\right)=\frac{\rho\left(\tilde{x}_{2}(t)\right)}{\alpha}$. Equation (10) shows that $V\left(\tilde{x}_{2}, \tilde{X}\right)=\frac{1}{2}\left(\frac{\rho\left(\tilde{x}_{2}\right)}{\alpha} \tilde{x}_{2}^{2}+\tilde{X}^{2}\right)$ is positive definite. Its time derivative along a trajectory of the system starting in $\tilde{D}^{+}$is

$$
\begin{aligned}
\dot{V} & =\frac{1}{2} \dot{P}\left(\tilde{x}_{2}\right) \tilde{x}_{2}^{2}+P\left(\tilde{x}_{2}\right) A\left(\tilde{x}_{2}\right) \tilde{x}_{2}^{2} \\
\dot{V} & =\frac{\rho\left(\tilde{x}_{2}\right)}{2 \alpha}\left[\left(1+\frac{\rho\left(\tilde{x}_{2}\right)}{\rho(0)}\right) A\left(\tilde{x}_{2}\right)+\frac{\rho\left(\tilde{x}_{2}\right)}{w_{g, i n}+\delta} \alpha X\right] \tilde{x}_{2}^{2}
\end{aligned}
$$

a) Negativeness of $\dot{V}$ : We now seek a sufficient geometric condition on $\left(\tilde{x}_{2}, X\right)$ to guarantee the negativeness of $\dot{V}$, i.e. we wish to determine a set $\Gamma$ in $\tilde{D}^{+}$containing the origin $\left(\tilde{x}_{2}, \tilde{X}\right)=(0,0)$, in which $\dot{V}\left(\tilde{x}_{2}, \tilde{X}\right)$ is negative. We will then build an invariant set $K$ inside $\Gamma$, such that every trajectory starting in $K$ will converge to the origin. More precisely, it is possible to construct a family of such sets $\Gamma$, which we denote $\Gamma^{\eta}$ where $\eta$ is a parameter to be chosen within a bounded interval. The construction is as follows. For any given $\eta>0$, we first seek a condition on $\tilde{x}_{2}$ so that

$$
A\left(\tilde{x}_{2}\right)<-\eta
$$

To include the case of the equilibrium point $\tilde{x}_{2}=0, \eta$ must be taken sufficiently small. Precisely, one must have

$$
A(0)=-\alpha+\beta-\frac{1}{c} \frac{\left(w_{g, i n}+\delta\right)^{2}}{\delta}<-\eta
$$

which is always consistent with the previous condition $\eta>$ 0 as it matches exactly the linear stability condition (6).
Further, a sufficient condition such that (12) holds can be easily determined. Two cases must be considered. If $\beta-\alpha \leq$ 0 , a right choice of $\eta$ yields an absence of restriction on $\tilde{x}_{2}$. Indeed, in this case, taking $0<\eta<\alpha-\beta+\rho_{\text {min }}$ yields, for all $\tilde{x}_{2} \in \tilde{D}^{+}, A\left(\tilde{x}_{2}\right) \leq-\eta<0$. Otherwise, if $\beta-\alpha>0$, then $A\left(\tilde{x}_{2}\right)$ is negative under the condition

$$
\tilde{x}_{2}>\left(c-\bar{x}_{2}\right)\left(\frac{-\alpha+\beta-\rho(0)+\eta}{-\alpha+\beta+\eta}\right)
$$

where $\eta$ can be freely chosen in the open interval $(0,-A(0))$. This condition defines a half-plane for $\left(\tilde{x}_{2}, \tilde{X}\right)$ which is now noted $\Gamma_{1}^{\eta}$. We now seek a sufficient condition for $\dot{V}$ to be negative in $\Gamma_{1}^{\eta}$, that is

$$
\alpha \frac{\rho\left(\tilde{x}_{2}\right)}{w_{g, i n}+\delta} \tilde{X}+\left(1+\frac{\rho\left(\tilde{x}_{2}\right)}{\rho(0)}\right) A\left(\tilde{x}_{2}\right)<0
$$

A sufficient condition is $\tilde{X}<\frac{1+\frac{\rho_{\min }}{\rho(0)}}{\alpha \frac{\rho \max }{w_{g, i n}+\delta}} \eta$ which can be rewritten as

$$
\tilde{X}<\frac{c \eta}{\alpha}\left(1+\frac{c-\bar{x}_{2}}{c-x_{2}^{*}}\right)
$$

This condition defines another half-plane which we note $\Gamma_{2}^{\eta}$. Gathering the previous inequalities, we have, for any $\eta>0$ satisfying (13)

$$
\forall\left(\tilde{x}_{2}, \tilde{X}\right) \in \Gamma^{\eta \stackrel{\text { def }}{=}} \tilde{D}^{+} \cap \Gamma_{1}^{\eta} \cap \Gamma_{2}^{\eta} \quad \dot{V}\left(\tilde{x}_{2}, \tilde{X}\right)<0
$$

b) Construction of an invariant set: For all $\eta, \Gamma^{\eta}$ contains the origin. Indeed, $\Gamma_{1}^{\eta}$ contains it thanks to (13), $\Gamma_{2}^{\eta}$ contains it because the upper bound on $\tilde{X}$ in (14) is positive, and $\tilde{D}^{+}$contains it also. Finally, $\Gamma^{\eta}$ is open as it is the intersection of three open sets containing the origin, therefore it contains a neighborhood of the origin. We take $\sigma=\min _{\left(\tilde{x}_{2}, \tilde{X}\right) \in \partial \Gamma^{\eta}} V\left(\tilde{x}_{2}, \tilde{X}\right)$, and $k<\sigma$. We define $K$ as

$$
K=\left\{\left(\tilde{x}_{2}, \tilde{X}\right) \in R^{2} \mid V\left(\tilde{x}_{2}, \tilde{X}\right) \leq k\right\}
$$

We claim that $K$ is a positively invariant compact set. First, notice that $K \subset \Gamma^{\eta}$. Indeed, $K \subset \Gamma^{\eta}$ because if $K \backslash \Gamma^{\eta} \neq \emptyset$, then $\exists\left(\tilde{x}_{2}, \tilde{X}\right) \in K \cap \partial \Gamma^{\eta}$. This would give $k \geq V\left(\tilde{x}_{2}, \tilde{X}\right) \geq \sigma>k$ which is impossible. This also shows that $K \cap \partial \Gamma^{\eta}=\emptyset$. Therefore, $K$ lies entirely in the interior of $\Gamma^{\eta}$.

Now, consider a trajectory starting in $K$. Suppose that there exists $t_{1} \geq 0$ such that $\left(\tilde{x}_{2}\left(t_{1}\right), \tilde{X}\left(t_{1}\right)\right) \notin K$. Then, the trajectories being continuous, there also exists $t_{2} \geq 0$ such that $\left(\tilde{x}_{2}\left(t_{2}\right), \tilde{X}\left(t_{2}\right)\right) \in \Gamma^{\eta} \backslash K$, and $\left(\tilde{x}_{2}(t), \tilde{X}(t)\right) \in \Gamma^{\eta}, \forall 0 \leq t \leq t_{2}$. Then, one would have $V\left(\tilde{x}_{2}\left(t_{2}\right), \tilde{X}\left(t_{2}\right)\right)>k \geq V\left(\tilde{x}_{2}(0), \tilde{X}(0)\right)$ which is impossible because $\dot{V}<0$ in $\Gamma^{\eta}$. Therefore, every trajectory starting in $K$ remains in $K$ for all future times.

c) Asymptotic stability: We now consider system (11) on the set $K$ defined by (16) and apply Lasalle's invariance principle with $V$ as a Lyapunov function. Indeed, $K$ is a non-empty compact set, and $\dot{V}\left(\tilde{x}_{2}, \tilde{X}\right)<0$ for all $\left(\tilde{x}_{2}, \tilde{X}\right) \in K$. Therefore, any trajectory starting in $K$ converges to the largest invariant set included in $\{x \mid \dot{V}(x)=0\}=$ $\left\{\left(\tilde{x}_{2}, \tilde{X}\right) \mid \tilde{x}_{2}=0\right\}$. Because $\alpha>0$, (11) shows that this set reduces to the origin. This shows that $K$ is included in the basin of attraction of the origin. 